\title{
Nachhaltige Dominanz? Präsident Bush, die Republikaner und ihr Masterplan
}

\author{
Hubert Silberhorn
}

Die Regierungszeit von George W. Bush wird als eine der kontroversesten Amtszeiten eines Präsidenten der Vereinigten Staaten von Amerika in die Geschichtsbücher eingehen. Nicht nur provoziert und polarisiert der 43. Präsident der USA die Demokraten und deren liberale Wähler mit einer kompromisslosen Kriegs- und Anti-Terrorstrategie; auch in der eigenen Partei und der konservativen Stammwählerschaft brodelt es beträchtlich. Dabei spielt weniger der verlustreiche, unilaterale und völkerrechtlich bedenkliche Waffengang im Irak eine Rolle als vielmehr die Abkehr der Bush-Administration von vormals sakrosankten republikanisch-konservativen Prinzipien. So erreichte die öffentliche Verschuldung im Jahre 2006 ein neues Rekordniveau ${ }^{1}$, und der Präsident betrieb unter dem Schlagwort des „compassionate conservatism“2 (mitfühlender Konservatismus) eine eigenwillige sozialpolitische Reformagenda, die insbesondere wegen der „spending explosion“ ${ }^{3}$ antithetisch zum Paradigma des „limited government" als „big government conservatism“ “4 bezeichnet wurde. Daher erscheint die These einer von Bush eingeleiteten anhaltenden Dominanz der Republikaner auch angesichts des für die Grand Old Party (GOP) katastrophalen Ausgangs der Zwischenwahlen 2006 geradezu paradox, zumal Bush, angetreten als „Uniter“5, das Ziel der gesellschaftlichen Einigung bei weitem verfehlte. Die USA unter und nach Bush sind und werden mehr gespalten sein als jemals zuvor - eine Spaltung jedoch mit einem kleinen, aber entscheidenden Vorteil für die Republikaner. Im Verlauf der Bush-Präsidentschaft wurde die „50-50 Nation“ auf Grund einer zielgerichteten Politik der Administration sowie

1 Im März 2006 hob der Kongress die Obergrenze der Staatsverschuldung zum vierten Mal in fünf Jahren auf nun neun Billionen US-Dollar an. Im Juli 2007 kündigte der Finanzminister an, dass im Herbst eine neuerliche Erhöhung nötig sein werde. Vgl. The Associated Press, Paulson Urges Congress To Raise U.S. Debt Limit, in: The Wall Street Journal Online vom 30. Juli 2007, Zugriff unter: http://online.wsj.com/article/SB118583405194882607.html (Zugriff am 20. August 2007).

2 Der Begriff entstand Bush selbst zufolge in einem Interview. Als der Reporter auf Bushs Aussage, er sei ein konservativer Politiker, erwiderte, dass seine Politiken dieser Bezeichnung nicht immer entsprechen würden, erwiderte Bush: „Well then, call me a conservative with a heart." Vgl. George W. Bush, A Charge to Keep, New York 1999, S. 235.

3 Vgl. Veronique de Rugy, The Republican Spending Explosion, Cato Brief Papers, Nr. 87, 3. März 2004, S. 3.

4 Vgl. Fred Barnes, Rebel-in-Chief. Inside the Bold and Controversial Presidency of George W. Bush, New York 2006, S. 159; Bruce Bartlett, Imposter. How George W. Bush Bankrupted America and Betrayed The Reagan Legacy, New York 2006, S. 2; Ryan Sager, The Elephant in the Room. Evangelicals, Libertarians and the Battle to Control the Republican Party, New Jersey 2006, S. 84; Jonathan Chait, Wages of Sin, in: The New Republic vom 22. Mai 2005, S. 22.

5 George W. Bush bezeichnete sich selbst wahlwirksam als ,uniter, not a divider“, vgl. John Harwood, GOP's New Campaign Themes Copied From Clinton Playbook, in: Pittsburgh Post-Gazette vom 18. Oktober 1998, S. A8. Zur verfehlten Zielsetzung, das Land zu einigen vgl. Fred I. Greenstein, The Man and His Leadership, in: Jon Fortier / Norman Ornstein (Hrsg.), Second-Term Blues. How George W. Bush has Governed, Washington DC 2007, S. 65. 
einer straff und professionell organisierten Wahlkampfführung zur „51-49 Nation“6. Dieser Vorsprung ist minimal, könnte aber die Basis für eine nachhaltige politische Dominanz der Republikaner bilden und ein neues Realignment einleiten.

\section{Der „compassionate conservatism“ und die programmatisch-philosophische Erneuerung}

Auf dem Weg, der Republikanischen Partei nachhaltig Mehrheiten zu sichern, spielte die Doktrin des compassionate conservatism insbesondere im Wahlkampf des Jahres 2000 eine zentrale Rolle. In den Reihen der Konservativen nicht unumstritten und zum Teil heftig kritisiert $^{7}$, umfasst diese Philosophie eine große Bandbreite sozial- und gesellschaftspolitischer Initiativen, rechtfertigt steigende Ausgaben und soll so das neue warmherzige Bild der Republikaner untermauern. „President Bush and Karl Rove have the vision, one they've promoted since Bush was governor: compassionate conservatism, a vision of rich and poor, and of whites, blacks and Hispanics, working together in communities and developing their own plans from the bottom up." ${ }^{8}$ Auf mehreren Ebenen hebt sich der mitfühlende Konservatismus von den bis zum Ende des 20. Jahrhunderts dominanten Leitlinien konservativer Gesellschafts- und Ordnungspolitik ab und bricht insbesondere mit der aggressiven antigouvernementalen Rhetorik Ronald Reagans und Newt Gingrichs. Bush selbst machte während seiner Kampagne klar, wie seine Vorstellungen moderner konservativer Regierungsphilosophie aussehen: „Conservatism has become the creed of hope. The creed of aggressive, persistent reform. The creed of social progress. [...]. Too often, my party has confused the

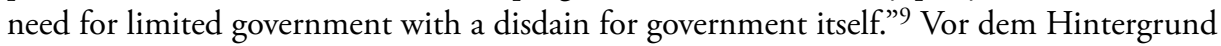
eines von innenpolitischen Themen dominierten Wahlkampfes im Jahr 2000 und der im Zeichen von „divided government“ durch Blockade geprägten Amtszeit Bill Clintons versprach die Strategie des mitfühlenden Konservatismus dem Wahlvolk einen pragmatischprogressiven und vor allem reformfähigen Regierungsstil, der wegen der gleichzeitigen Betonung des individuellen Verantwortungsbewusstseins und der damit verbundenen moralischen Erneuerung der Gesellschaft die konservative Wählerschaft ansprach.

6 Vgl. Michael Barone, Introduction, in: ders. / Richard E. Cohen (Hrsg.), The Almanac of American Politics 2006, Washington DC 2005.

7 Im Jahre 1999 lehnten damalige Republikanische Parteigrößen von Gouverneur Lamar Alexander über Steve Forbes bis hin zu Dan Quayle den Begriff strikt ab. Alexander: „Is Mr. Bush trying to tell us that he's not like the rest of us conservatives? That conservatism, as a political philosophy, is by definition without compassion?" Zitiert in: Andy Sher, Alexander taunts peers for coining new phrases, in: Arkansas Democrat-Gazette vom 22. Januar 1999, S. A7. Steve Forbes: „Mealymouthed rhetoric and poll-tested clichés [are] no substitute for a muscular, substantive agenda.” Zitiert in: Commentary, Republican rivals seem to boost Gore, in: Tampa Tribune vom 7. Februar 1999, S. 6. Dan Quayle: „This silly and insulting term was created by liberal Republicans and is nothing more than code for surrendering our values and principles." Zitiert in: John Micklethwait I Adrian Woolridge, The Right Nation, Conservative Power in America, New York 2004, S. 101.

8 Vgl. Marvin Olasky, Karl Rove Time, in: Townhall.com, 13. November 2002, Zugriff unter: http://www.townhall.com/columnists/MarvinOlasky/2002/11/13/karl_rove_time (Zugriff am 8. Mai 2007).

9 Vgl. George W. Bush, A Culture of Achievement, The Future of Educational Reform, The Manhattan Institute for Policy Research, 5. Oktober 1999, Zugriff unter: http://www.manhattan-institute.org/html/bush_speech.htm (Zugriff am 30. April 2006). 
Einerseits lehnt diese Programmatik staatliche Interventionen beziehungsweise die Rolle des Staates nicht per se $\mathrm{ab}$ und nimmt daher in begrenzten Bereichen auch steigende Ausgaben in Kauf. Andererseits basiert der mitfühlende Konservatismus auf einer strikten Ziel- und Ergebnisorientierung, die die staatliche Rolle an Resultate knüpft. Zudem werden kulturelle, genuin amerikanische Werte betont, wie etwa die besondere Rolle der „communities" und der kirchlich-religiösen Einrichtungen; man wendet sich gegen den paternalistischen Staat und dessen "gemeinschaftsschädlichen" Auswüchse und setzt auf die Förderung des Individuums und seiner spezifischen Fähigkeiten. Solche Positionen spiegeln eindeutig neo-konservative Vorstellungen wider. $^{10}$

Im Hinblick auf konkrete Politiken zeigt sich, dass der „mitfühlende Konservatismus“ keineswegs einen Staat im Sinne von Lyndon B. Johnsons „Great Society“ anzielt. Vielmehr ist die Konzeption als im Kern strukturkonservativ zu verstehen, die mit ihrer Programmatik geplant auf ausgewählte, für zukünftige Wahlerfolge entscheidende Wählerschichten zugeht und damit alte Konfliktlinien durchbricht beziehungsweise zum Vorteil der Republikaner aufweicht. Karl Rove, der im Sommer 2007 zurückgetretene einflussreichste Berater in der Bush-Administration und Architekt der Strategie, unterstrich die Notwendigkeit neuer Konzeptionen und erhellte damit nochmals die Bedeutung des compassionate conservatism: „I think we're at a point where the two major parties have sort of exhausted their governing agendas. [...] somebody [die Bush-Administration] will come along and figure out a new governing scheme through which people could view things and could, conceivably, enjoy a [new] period of dominance." 11 Der mitfühlende Konservatismus ist der philosophisch-programmatische Rahmen und damit der von Rove erwähnte neue Ansatz des Regierens, der Politiken ermöglicht, die gleichzeitig traditionelle Wähler und die für den politischen Erfolg so wichtigen Wechselwähler ansprechen. Das Konzept kann somit als Weg verstanden werden, den US-Konservatismus an die Ära nach dem Kalten Krieg anzupassen. ${ }^{12}$

\section{Die neue Minderheitenpolitik und das Ende der "southern strategy“}

Aus historischer Sicht ist die Minderheitenpolitik der Republikanischen Partei durchaus ambivalent. Etablierten sich die frühen Republikaner im Bürgerkrieg und den folgenden Dekaden als die Partei der schwarzen Minderheit, setzte insbesondere im Zuge der Bürger-

10 Die neo-konservative Bewegung, die ihren Ursprung in den 1970er Jahren hat, zielt mit ihrer Kritik besonders auf einen staatlich (demokratisch) geförderten und geforderten Egalitarismus ab, der sich in steigenden staatlichen Wohlfahrtsleistungen ausdrückt und essentielle kulturelle und moralische Grundwerte verdrängt. Dieser „demokratische Exzess” unterminiere jedoch die Demokratie selbst. „Yet the danger of overloading the political System with demands which extend its functions and undermine its authority still remains. The absence of such values [das Erbe traditioneller und aristokratischer Werte] in the United States produces a lack of balance in the society." Samuel P. Huntington, The United States, in: Michel Crozier / Samuel P. Huntington / Joji Watanuki (Hrsg.), The Crisis of Democracy, Report to the Trilateral Commission, New York 1975, S. 59 - 119, S. 114; Micklethwait und Woolridge sprechen in diesem Zusammenhang von "liberal social engineering", vgl. dies., a.a.O., S. 260.

11 Nicholas Lemann, The Controller, in: The New Yorker vom 12. Mai 2003, S. 75.

12 Vgl. John D. McKinnon / Jackie Calmes, After Rove Strategy Choices Loom, in: The Wall Street Journal vom 14. August 2007, S. A3. 
rechtsdebatte der 1960er Jahre ein Wandel ein, aus dem die Demokraten als die Partei der Minderheiten hervorgingen. Die Republikaner hingegen verfolgten eine Strategie, die in der mehrheitlich weißen Bevölkerung der südlichen Bundesstaaten Ängste vor Überfremdung schürte und inhaltlich immer wieder durch eine kompromisslose Haltung in Immigrationsfragen auf sich aufmerksam machte. ${ }^{13}$

Diese so genannte southern strategy sollte sich jedoch zum Ende des 20. Jahrhunderts grundlegend ändern, wobei einer kleinen Gruppe von Republikanern, allen voran George W. Bush und dessen Bruder Jeb, eine maßgebliche Rolle zukommt. Beide Politiker verfolgten als Gouverneure in Texas beziehungsweise Florida einen neuen Weg, um der sich rasch ändernden demographischen Entwicklung Rechnung zu tragen: Die USA verzeichnen einen stetigen Zuwachs der hispanischen Bevölkerung, die sich allein zwischen 1990 und 2000 mehr als verdoppelte und 2005 14,5 Prozent der US-Bevölkerung entsprach. ${ }^{14}$ „Hispanics are potentially the fastest-growing segment of the electorate - [...] $17 \%$ of the population under age 18 , between $6 \%$ and $8 \%$ of the 2004 electorate." ${ }^{15}$ Somit wird klar, welch immer wichtigere elektorale Rolle den lateinamerikanischen Einwanderern insbesondere in den „battleground states“ zukommt, den Schlüsselstaaten, wo die Wahlen entschieden werden. Diese Gruppe für sich zu gewinnen stellt für die Republikaner daher eine existenzielle Herausforderung dar, um nicht über Jahre hinweg ins politische Hintertreffen zu geraten.

Bush erkannte dies zu einem frühen Zeitpunkt und propagierte bereits als Gouverneur eine damals für die Republikaner fast unerhörte Offenheit. So kritisierte der Texaner die English-Only-Bewegung und setzte sich stattdessen für English-Plus ein ${ }^{16}$, wandte sich öffentlich gegen den kalifornischen Gouverneur und „presidential hopeful“ Pete Wilson und dessen „Proposition 187“, beförderte mit Alberto Gonzales einen „Hispanic“ in den texanischen Verfassungsgerichtshof, setzte eine Bildungsreform durch, von der sich besonders die Bevölkerungsminderheiten Vorteile erhofften und schlug einen Ton an „that says to the Hispanic Community, you're part of the future" 17 . So entstand ein Bild, das dem Gouver-

13 Noch in den 1990er Jahren propagierten die Republikaner auf nationaler wie auch auf einzelstaatlicher Ebene diese Politik. 1994 trat der kalifornische Gouverneur Pete Wilson vehement für die Proposition 187 ein, die illegalen Einwanderern den Zugang zur medizinischen Versorgung und zu öffentlichen Schulen verweigerte. Im Jahre 1996 zog der Kongress nach und verabschiedete Maßnahmen, die selbst legalen Immigranten staatliche Sozialleistungen verweigerten. Vgl. Tom Hamburger / Peter Wallsten, One Party Country. The Republican Plan for Dominance in the 21st Century, Hoboken, New Jersey 2006, S. 72.

14 Vgl. US Census Bureau, Population by Race and Hispanic or Latino Origin for the United States: 1990 and 2000, Table 4, 2. April 2001, Zugriff unter: http://www.census.gov/population/www/ cen2000/phc-t1.html (Zugriff am 20. August 2007); vgl. PEW Hispanic Center, A Statistical Portrait of Hispanics at Mid-Decade, Table 1, 18. September 2006, Zugriff unter: http://pewhispanic.org/reports/middecade/ (Zugriff am 20. August 2007).

15 Vgl. Michael Barone, a.a.O., S. 27.

16 Vgl. Gary Martin, Governors give Bush a push, in: San Antonio Express-News vom 22. Februar 1999, S. A1; George W. Bush, A Charge to Keep, a.a.O. Dieser Ansatz wird von der religiösen Rechten sowie den traditionell-Konservativen scharf kritisiert. Siehe dazu Patrick J. Buchanan, State of Emergency, The Third World Invasion and Conquest of America, New York 2006; Richard Viguerie, Conservatives Betrayed. How George W. Bush and Other Big Government Republicans Hijacked the Conservative Cause, Los Angeles 2006, S. 51 - 71; Newt Gingrich, To Renew America, New York 1995, S. 159 - 162.

17 Vgl. George W. Bush, zitiert in: Scott Lindlaw, George W. Bush Courts Hispanic Vote, Associated Press vom 28. Juni 1999. Zur Proposition 187 vgl. Fn. 12. 
neur die Unterstützung von 46 Prozent der hispanischen Bevölkerung in der Wahl 1998 einbrachte und das der Präsidentschaftskandidat Bush erfolgreich in den Wahlkampf und später ins Weiße Haus transportierte. Bezeichnend ist dabei, wie weit die Parteiplattformen aus den Jahren 1996 und 2000 in der Minoritäten- und Einwanderungsfrage auseinander liegen. 1996 wurde von einer Krisensituation der illegalen Einwanderung gesprochen und ein hartes Vorgehen angemahnt. ${ }^{18}$ Die 2000er Plattform hingegen wollte eine Botschaft für alle US-Bürger bringen, ,particularly immigrants and minorities: this is the party of freedom and progress, and it is your home" ${ }^{19}$.

Schon in den Präsidentschaftswahlen 2000 zahlte sich der neue Kurs aus. Hatte Clinton gegenüber Dole noch einen Vorsprung von 51 Prozentpunkten bei den hispanischen Wählern, konnte Bush den Rückstand auf 27 Prozentpunkte verkürzen. Ein noch besseres Ergebnis erzielte der Republikaner 2004, als der Abstand nunmehr neun Prozentpunkte betrug (vgl. Tabelle 1). ${ }^{20}$

\begin{tabular}{|l|l|l|}
\hline \multicolumn{2}{|c|}{ Tabelle 1: „Hispanic Vote“ in den US-Präsidentschaftswahlen 1996 bis 2004 } \\
\hline & Republikaner & Demokraten \\
\hline 1996 & $21 \%$ (Bob Dole) & $72 \%$ (Bill Clinton) \\
\hline 2000 & $35 \%$ (George W. Bush) & $62 \%$ (Al Gore) \\
\hline 2004 & $44 \%$ (George W. Bush) & $53 \%$ (John Kerry) \\
\hline
\end{tabular}

Quelle: Voter News Service/National Election Pool, CNN Exit Poll, Zugriff unter: http://www.cnn.com/ ALLPOLITICS/1996/elections/natl.exit.poll/index1.html; http://www.cnn.com/ELECTION/2000/results/index.epolls.html; http://www.cnn.com/ELECTION/2004/pages/results/states/US/P/00/epolls.0. html (Zugriff am 20. März 2007).

Noch deutlicher wird der Stellenwert der hispanischen Wähler bei einem Blick auf die Einzel- und dabei besonders auf die Schlüsselstaaten. Geographisch sind die Wahlpräferenzen in den USA relativ klar verteilt. Die Demokraten gewinnen regelmäßig die Bundesstaaten an den Küsten, während die Republikaner den Süden und den Westen beherrschen. ${ }^{21}$ Schon vor den Wahlen steht somit weitgehend fest, welche Bundesstaaten für den Wahlausgang kritisch sein werden. 2004 galten 181 Stimmen des Wahlmännergremiums als sicher für Bush, 168 Stimmen für Kerry. In den umkämpften Staaten waren weitere 189 Stimmen zu vergeben, wobei 270 für den Wahlsieg notwendig sind. Dabei spielen einige Staaten mit einer signifikanten hispanischen Minderheit eine herausragende Rolle. Wie Tabelle 2 zeigt, konnte Bush sein Wahlergebnis von 2000 analog zur steigenden Zustimmung der Hispanics, mit Ausnahme von Colorado, in allen Staaten mit einem hispanischen Anteil von über 15 Prozent der Gesamtbevölkerung verbessern, wobei fünf dieser Staaten als Schlüssel-

18 Vgl. Preamble, Republican Platform 1996, Zugriff unter: http://www.cnn.com/ELECTION/2000/conventions/republican/features/platform.96/ (Zugriff am 15. April 2007).

19 Vgl. Preamble, Republican Platform 2000, Zugriff unter: http://www.cnn.com/ELECTION/2000/conventions/republican/features/platform.00/ (Zugriff am 15. April 2007).

20 Das Ergebnis des Jahres 2004 stellte den geringsten Abstand zu den Demokraten seit Beginn der „Exit Polls“ im Jahre 1972 dar. Vgl. Thomas B. Edsall, Building Red America, The New Conservative Coalition and the Drive for Permanent Power, New York 2006, S. 219.

21 Vgl. Gerald M. Pomper, The 2000 Presidential Election, Why Gore Lost, in: Political Science Quarterly, 116. Jg. (2001), H. 2, S. $201-204$. 
staaten gelten. In New Mexico gelang es Bush sogar, knapp zwölf Prozent der hispanischen Wähler hinzuzugewinnen, was wesentlich dazu beitrug, dass der Staat den Demokraten verloren ging. ${ }^{22}$ Dabei ist jedoch festzuhalten, dass die Strategie der Administration keineswegs eine unrealistische mehrheitliche „Übernahme“ der hispanischen Minderheit bezweckte, sondern sich vielmehr darauf konzentrierte, den Anteil in den „swing states“, den Staaten mit wechselnden Mehrheiten, moderat und abgesichert zu erhöhen, und so den entscheidenden Vorteil in den stark polarisierten Schlüsselstaaten zu erzielen. „Some 70.000 votes among Colorado, Nevada, and New Mexico, with their collective 19 electoral votes, would have swung the presidential election [2004] just as surely as Ohio's 20 electoral votes." 23

Diese Strategie wird noch plausibler angesichts demographischer Migrationsbewegungen innerhalb der USA. Die US-Bevölkerung wandert verstärkt in den so genannten Sun-Belt - die Staaten des Südens und des Westens - ab. Im Zuge dieser Migration werden Nevada $(+2)$, New Mexico (+5), Kalifornien (+2), Texas (+8) und Florida (+9) bis zum Jahre 2030 einen höheren Stimmenanteil im Wahlmännerkollegium erhalten. Der Stimmenvorteil der südlichen Staaten gegenüber jenen des Nordens, dem Snow Belt, wird so von 88 Stimmen auf 146 ansteigen. Würde das Wahlergebnis des Jahres 2004 in das Jahr 2030 transponiert werden, zöge dies massive Vorteile für die Republikaner nach sich. ${ }^{24}$

\begin{tabular}{|c|c|c|c|c|c|c|}
\hline \multicolumn{7}{|c|}{$\begin{array}{l}\text { Tabelle 2: Demographische Werte und Wablverhalten der hispanischen Minderheit in den USA } \\
2000 \text { und } 2004 \text { (Angaben in Prozent) }\end{array}$} \\
\hline & $\begin{array}{l}\text { Stimmen im } \\
\text { Wahlmän- } \\
\text { nergremium }\end{array}$ & $\begin{array}{l}\text { Anteil der } \\
\text { Hispanics an } \\
\text { der Bevölke- } \\
\text { rung } 2000\end{array}$ & $\begin{array}{l}\text { Bushs Wahl- } \\
\text { ergebnis } \\
2004\end{array}$ & $\begin{array}{l}\text { Bushs Wahl- } \\
\text { ergebnis } \\
2000\end{array}$ & $\begin{array}{l}\text { Stimmen der } \\
\text { hispanischen } \\
\text { Wähler } 2004\end{array}$ & $\begin{array}{l}\text { Stimmen der } \\
\text { hispanischen } \\
\text { Wähler } 2000\end{array}$ \\
\hline Arizona & $10^{*}$ & 25,3 & 55 & 51 & 43 & 27,0 \\
\hline Kalifornien & 55 & 32,4 & 45 & 42 & 32 & 27,5 \\
\hline Colorado & $9^{*}$ & 17,1 & 52 & 51 & 30 & 33,0 \\
\hline Florida & $27^{*}$ & 16,8 & 52 & $49^{* *}$ & 56 & 49,4 \\
\hline Nevada & $5^{*}$ & 19,7 & 51 & $49^{* *}$ & 39 & n.a. \\
\hline Texas & 34 & 32,0 & 61 & 59 & 49 & 43 \\
\hline \multirow[t]{2}{*}{ New Mexico } & $5^{*}$ & 42,1 & $50^{* *}$ & 48 & 44 & 32,2 \\
\hline & 145 & & & & & \\
\hline \multicolumn{7}{|c|}{$\begin{array}{l}\text { * battleground states (Schlüsselstaaten). } \\
\text { ** gewonnener Staat. } \\
\text { Quelle: US Census Bureau, Hispanic or Latino Origin for the United States, Regions, Divisions, States, } \\
\text { and for Puerto Rico: } 2000 \text { (PHC-T-10), Zugriff unter: http://www.census.gov/population/www/cen2000/ } \\
\text { phc-t10.html; National Election Pool, CNN Exit Poll } 2000 \text { und 2004, NBC Exit Poll 2004, Zugriff } \\
\text { unter: http://www.cnn.com/ELECTION/2000/results/index.epolls.html; http://www.cnn.com/ELEC- } \\
\text { TION/2004/pages/results/states/US/P/00/epolls.0.html; http://www.msnbc.msn.com/id/5297118/; (Zu- } \\
\text { griff am 20. März 2007). }\end{array}$} \\
\hline
\end{tabular}

22 Vgl. Tom Hamburger / Peter Wallsten, a.a.O., S. 163.

23 Vgl. Ryan Sager, a.a.O., S. 163.

24 „Such shifts portend the continued decline in electoral power of blue Snow Belt states, and large gains by red Sun Belt states." William H. Frey, The Electoral College Map moves to the Sun Belt, Metropolitan Policy Program, The Brookings Institution, Mai 2005, S. 4, Zugriff unter: http:// www.brookings.edu/metro/pubs/20050504_electoralcollege.htm (Zugriff am 22. August 2007). 
Neben noch zu diskutierenden spezifischen Politiken erklärt auch die persönliche Haltung des Präsidenten zu Immigrationsfragen dessen gute Wahlergebnisse. Bereits zu Beginn seiner Amtszeit setzte sich Bush für eine umfassende Reform des Einwanderungsrechts inklusive eines Gastarbeiterprogramms ein - ein Vorhaben, das jedoch auf Grund der neuen Sicherheitslage nach dem 11. September 2001 in den Hintergrund geriet. ${ }^{25} 2004$ kehrte das Thema zurück auf die Tagesordnung in Washington DC, scheiterte aber am vehementen Widerstand konservativer Abgeordneter im Repräsentantenhaus um Tom Tancredo (Colorado), die den Vorschlag als „Amnestie“ bezeichneten. Der Gesetzentwurf von John McCain (Rep.) und Edward Kennedy (Dem.) sah nämlich ein Bleiberecht für illegale Immigranten und vereinfachte Einreiseregelungen vor. ${ }^{26}$ Wiederum konnte das Vorhaben nicht verwirklicht werden und offenbarte der Republikanischen Partei zugleich die erheblichen ideologischen Probleme und das enorme Konfliktpotenzial des gesellschaftspolitisch aufgeladenen Einwanderungsthemas. Wahltaktisch wirkte sich die fortgesetzte Unfähigkeit, eine umfassende Einwanderungsreform zu realisieren, umgehend aus. Die immigrationsfeindliche Haltung einiger republikanischer Abgeordneter führte zu einem signifikanten Rückgang der hispanischen Stimmen in den Zwischenwahlen 2006, als nur noch 29 Prozent die Republikaner unterstützten. "They're caught between a passionate minority of their party who oppose any reform that allows illegals a path to citizenship - and the larger electorate, which is more moderate and wants to solve the problem." ${ }^{27}$

Auch der letzte Versuch der Bush-Administration und ihrer verbündeten Kongressmitglieder, wie Jon Kyle (Rep., Arizona), Mike Pence (Rep., Indiana) und Edward Kennedy (Dem., Massachusetts), scheiterte im Sommer 2007 hauptsächlich an der eigenen Partei, aber auch an der demokratischen Führungsriege im Senat. Der Präsident, der in der zweiten Amtszeit bisher keinen großen legislativen Erfolg zu verbuchen hatte, mahnte seine Partei eindringlich: „If you're viewed as anti - in other words, if people think that a party is against somebody or some group of people, you'll pay a political price for it. [...] If you're viewed as anti-immigrant, [....] it could cause serious long-term political consequences." 28 Diese Einsicht bewog letztlich auch die demokratische Führung um Senator Harry Reid (Nevada), keine Kompromisse einzugehen. Zu viel wahltaktisches Potenzial liegt in der Immigrationsfrage, zu hoch scheint das politische Risiko, dem Präsidenten einen wesentlichen legislativen Erfolg zuzugestehen, zu verlockend die Aussicht, im Wahlkampf 2008 den Präsidenten und damit dessen Partei als gescheitert zu porträtieren. „Assuming the measure passes in Congress - a dicey assumption at this point - it should save Republicans from further erosion of support among Hispanics. This is crucial to Republican prospects for holding the White House in 2008 and recapturing Congress. And it may give Bush, long an advocate of immigration reform, a political boost and jack up his approval rating." 29

Obgleich die Republikaner unter Bush ihre Wahlchancen bei der hispanischen Minderheit und damit die Ausgangsposition bei Präsidentschafts- und Kongresswahlen massiv ver-

25 Vgl. Bruce Bartlett, a.a.O., S. 206; John Micklethwait / Adrian Woolridge, a.a.O., S. 241.

26 Vgl. John B. Judis, Border War, in: The New Republic vom 16. Januar 2006, S.19.

27 Review \& Outlook, Immigration and the GOP, in: The Wall Street Journal vom 27. Juni 2007, S. A12.

28 George W. Bush, zitiert in: Kimberley Strassel, Latin Lessons, in: The Wall Street Journal vom 1. Juni 2007, S. A12.

29 Fred Barnes, Bordering on Progress, in: The Weekly Standard vom 28. Mai 2007, S. 16. 
bessern konnten, darf dies nicht darüber hinwegtäuschen, dass die Partei in der Immigrationsfrage tief gespalten ist. Das Verhältnis der hispanischen Minderheit zur GOP wird daher maßgeblich von der Kandidatenkür 2008 und der zukünftigen Haltung der Partei in Immigrationsfragen abhängen. Fest steht jedoch, dass Bushs Minderheitenpolitik seine Wahlerfolge begünstigte, wenn nicht sogar erst ermöglichte. Auf dem Weg zu einem neuen „realignment“ und langfristiger republikanischer Dominanz spielt die hispanische Minderheit eine entscheidende Rolle.

\section{3. "The Politics of Faith"}

Religion, Werte und Moral spielen in der US-Gesellschaft eine zunehmend wichtige Rolle, die allmählich auch auf die Politik ausgreift. Im Jahre 2000 besuchten 58 Prozent der USBürger mindestens einmal im Monat einen Gottesdienst. ${ }^{30}$ Hinzu kommt, dass sich 80 Prozent der Amerikaner als Christen bezeichnen. ${ }^{31}$ Der hohe Stellenwert der Religiosität in der Gesellschaft wirkt sich insofern politisch aus, als auch von Kandidaten und Mandatsträgern beziehungsweise den Parteien allgemein eine gewisse Moralität und religiöses Empfindungsvermögen erwartet werden. Immerhin gaben im Jahre 200070 Prozent der Wähler an, sie wünschten sich „a person of faith“ als ihren Präsidenten ${ }^{32}$, und 2004 gaben 80 Prozent der Bush-Wähler an, moralische Werte seien für ihre Wahlentscheidung ausschlaggebend gewesen. ${ }^{33}$ Dennoch dürfen diese Zahlen nicht darüber hinwegtäuschen, dass zwei Drittel der US-Bürger eine aktive Einmischung der Kirchen in parteipolitische Fragen ablehnen. ${ }^{34}$ In religiösen Fragen steht somit für die Parteien viel auf dem Spiel - die Haltung zu Moral und gesellschaftlichen Grundwerten wird zu einem politischen Drahtseilakt, den beide Parteien zu meistern haben und dessen Ausgestaltung ebenso umstritten ist.

Die Republikaner zählen seit der Präsidentschaft Ronald Reagans die religiöse Rechte zu ihren wichtigsten Unterstützern. 2004 wählten 78 Prozent der weißen Evangelikalen George W. Bush. ${ }^{35}$ Zudem gewann er die Mehrheit der Protestanten (59 Prozent) sowie der Ka-

30 Vgl. Voter News Service Exit Poll, 2000 Zugriff über CNN unter: http://www.cnn.com/ELECTION/2000/results/index.epolls.html (Zugriff am 3. Juli 2007).

31 Vgl. Josef Braml, Die theo-konservative Politik Amerikas, in: APuZ, B 7 (2005), S. 30.

32 Vgl. Pew Research Center for the People \& the Press, Religion and Politics, The ambivalent Majority, 20. September 2000, Zugriff unter: http://people-press.org/reports/display.php3?ReportID $=32$ (Zugriff am 25. Juli 2007).

33 Vgl. David W. Moore, Moral Values Important in the 2004 Exit Polls, Gallup Poll News Service, 7. Dezember 2004. Im Jahre 2001 umschrieb ein Bewohner des ländlichen Pennsylvania seine Entscheidung, für Bush zu wählen, folgendermaßen: „Rural America is pissed, these people are tired of moral decay. They are tired of everything being wonderful on Wall Street and terrible on Main Street." Diese Aussage führte den Autor Thomas Frank zu der erstaunlichen Schlussfolgerung: „They're voting Republican in order to get even with Wall Street.” Thomas Frank, What's the Matter with Kansas? How Conservatives Won the Heart of America, New York 2004, S. $23 \mathrm{f}$.

34 Vgl. Pew Research Center for the People \& the Press, Faith-Based Funding Backed, But ChurchState Doubts Abound, Introduction and Summary, 10. April 2001, Zugriff unter: http://peoplepress.org/reports/display.php3?PageID=111 (Zugriff am 26. Juli 2007).

35 Vgl. CNN Exit Poll 2004, Zugriff unter: http://www.cnn.com/ELECTION/2004/pages/results/ states/US/P/00/epolls.0.html (Zugriff am 20. März 2007). 
tholiken (52 Prozent) für sich und baute die „moral majority“ im Vergleich zum Jahre 2000 weiter aus. So konstituiert die Religion eine der klarsten Konfliktlinien in der modernen US-Gesellschaft. ${ }^{36}$ Die Mahnung der konservativen Ikone Barry Goldwater scheint dabei keine Rolle mehr zu spielen: „One of the strengths of our political system always has been our tendency to keep religious issues in the background. " 37

In diesem Zusammenhang zeigt sich die Rhetorik des mitfühlenden Konservatismus als wesentlicher Baustein der moralisch behafteten Politik der Bush-Administration. Eine besondere Rolle kommt dabei den so genannten Faith Based Initiatives zu - religiös-karitative Einrichtungen, die an Stelle des Staates, jedoch mit dessen finanzieller Hilfe, Aufgaben im sozialen Bereich wahrnehmen. „These institutions, at their best, treat people as moral individuals, with responsibilities and duties, not as wards or clients or dependents or numbers." 38 Faith Based Initiatives sind die Mittel des mitfühlenden Konservatismus, mit deren Hilfe konservative Attribute wie Eigenverantwortlichkeit, Religiosität und Gemeinschaftssinn revitalisiert werden und der fortschreitenden „Liberalisierung“ des öffentlichen und zivilen Lebens, die sich in dieser Sicht durch eine staatliche Förderung im Rahmen von „welfare" auszeichnete und Abhängigkeiten förderte, entgegengestellt werden. Faith Based Organizations (FBOs) bilden das institutionalisierte Kernstück des compassionate conservatism und geben die große politische Flexibilität des Konzepts wieder als „something [that] will be aimed simultaneously at both base voters, on the right, and swing voters, in the middle“39.

Seit FBOs durch die „welfare“-Gesetzgebung aus dem Jahre 1996 offiziell als „government proxies", also anstatt staatlicher Stellen fungieren können, keimen zwar immer wieder Fragen nach der konstitutionellen Rechtmäßigkeit dieser Regelung auf ${ }^{40}$, gleichzeitig hat sich aber ein gesellschaftlich-überparteilicher Konsens zur Rolle religiöser Gruppierungen und sozialer Aufgabenübernahme etabliert. Im September 2001 befürworteten 74 Prozent der Demokraten eine staatliche Förderung religiös-karitativer Einrichtungen. Gleichzeitig sprachen sich zu diesem Zeitpunkt „nur“ 63 Prozent der Republikaner für derartige Initiativen aus. Die Demokraten waren lediglich hinsichtlich der Effektivität dieser Gruppen unschlüssig: 33 Prozent waren der Ansicht, religiöse Gruppen würden zu besserer Arbeit imstande sein als der Staat. ${ }^{41}$ Hier wird deutlich, welch großen Stellenwert religiös-mora-

36 Vgl. Michael Barone, a.a.O., S. 26.

37 Excerpts of the Goldwater Remarks, in: The New York Times vom 16. September 1981, S. B9.

38 George W. Bush, The Duty of Hope, Rede in Indianapolis am 22. Juli 1999.

39 Nicholas Lemann, a.a.O.

40 Vgl. Angie Welborn, Charitable Choice: Legal and Constitutional Issues, CRS Report for Congress, 27. Januar 2006; Emily R. Gill, Religious Organizations, Charitable Choice, and the Limits of Freedom of Conscience, in: Perspective on Politics, 2. Jg. (2004), S. 741 - 756; vgl. Marc D. Stern, Charitable Choice: The Law As It Is and May Be, in: Andrew Walsh (Hrsg.), Can Charitable Choice Work? Covering Religion's Impact on Urban Affairs and Social Services, Hartford, CT 2001; ausführlich zu Charitable Choice vgl. Joe Richardson, Charitable Choice Rules and Faith-Based Organizations, CRS Report for Congress, 24. März 2005, sowie zu den Prinzipien von Charitable Choice John Dilulio, Government by Proxy: A Faithful Overview, in: Harvard Law Review, 116. Jg. (2003), H. 5, S. 1278.

41 Vgl. Pew Research Center for the People \& the Press, Faith-Based Funding Backed, But ChurchState Doubts Abound, Section I, Funding for Faith-Based Organizations: Broader Support, Deeper Differences, 10. April 2001, Zugriff unter: http://people-press.org/reports/display. php3?PageID=111 (Zugriff am 26. Juli 2007). 
lische Themen selbst für die gesellschaftlich-liberal orientierten Demokraten aufweisen. ${ }^{42}$ Zudem untermauern diese Zahlen die taktische Klugheit des mitfühlenden Konservatismus und der auf religiösem Glauben beruhenden Agenda der Administration, die trotz ihres weitgehenden Scheiterns als „cross around the White House’s neck“ 43 erschienen und somit die moralische Mehrheit ebenso ansprachen wie sozial-konservative Demokraten.

In diesem Zusammenhang ist auf das große Potenzial dieser Politik für eine Wählergruppe zu verweisen, die den Republikanern in der Vergangenheit vernichtende Wahlergebnisse einbrachte: die Afroamerikaner. Diese sehen nach wie vor die „southern strategy“ sowie die Absorption früherer Befürworter der Rassentrennung als Ausdruck des rassistischen Grundgedankens der GOP. Im Jahre 2000 wählten lediglich acht Prozent der Afroamerikaner den Kandidaten Bush. Erklärtes Ziel war es, diesen geringen Anteil auszubauen und dabei ähnlich wie im Falle der hispanischen Bevölkerung die gesamten Bemühungen auf den „reformierbaren“ Teil der Afroamerikaner zu konzentrieren, denn „in a close election, reducing black support for Democratic candidates by even a few percentage points could make a difference“ 44 .

Die Chancen schienen gut zu stehen, da insbesondere Afroamerikaner Faith Based Initiatives mit überwältigender Mehrheit unterstützen. 81 Prozent der schwarzen Amerikaner sprachen sich 2001 für eine Förderung der FBOs aus. ${ }^{45}$ Bushs Eintreten für „charitable choice“ und die politisch-institutionelle Unterstützung der FBOs darf daher nicht als rein religiös-idealistisches Vorgehen gesehen werden - eine Etikette, die oftmals vorschnell vergeben wird -, sondern auch als Wahlstrategie. So wurde das Thema Faith Based Initiatives im Wahlkampf 2002 wie auch 2004 gezielt als politische Waffe eingesetzt. Die Zielgruppe waren dabei insbesondere afroamerikanische Gemeinden, die sich bisher bei der Zuteilung staatlicher Fördergelder benachteiligt fühlten und nun von der glaubensfundierten Agenda der Republikaner maßgeblich profitieren sollten.

Vertreter des „Office for Faith Based and Community Initiatives“ (OFBCI) wurden in besonders umkämpfte Distrikte und für die Präsidentschaftswahl entscheidende battleground states gesandt, um Seminare und Kundgebungen abzuhalten, um die Initiative des Präsidenten und potenzielle Fördermöglichkeiten zu erläutern. Zum Beispiel wurden zu einer Veranstaltung, getragen von der lokalen Republikanischen Partei in South Carolina, wo nach dem Tode Strom Thurmonds Senatswahlen anstanden, 1600 schwarze Seelsorger eingeladen. Der politische Direktor der Republikaner in South Carolina, Ron Thomas, sprach im Anschluss daran von einem „phänomenalen Erfolg, der geholfen habe, der Partei

42 Auch Hillary Clinton trug dieser Tatsache Rechnung und sprach sich deutlich für FBOs aus: „I have always been a praying person. There is no contradiction between support for faith-based initiatives and upholding our constitutional principles." Zitiert in: Michael Jonas, Sen. Clinton urges Use of Faith-Based Initiatives, in: The Boston Globe vom 20. Januar 2005, S. B1.

43 David Kuo, Please Keep Faith, in: Beliefnet.com vom 14. Februar 2005, Zugriff unter: http:// www.beliefnet.com/story/160/story_16092_1.html (Zugriff am 15. August 2007). Kuo war vormals Berater der Bush-Administration. Trotz mehrfacher Versuche, die Reichweite der Faith Based Initiatives durch ein legislatives Fundament auszudehnen, scheiterten die Republikaner mehrfach im Kongress. Der gegenwärtige Handlungsspielraum der FBOs basiert daher lediglich auf einer Reihe von „Executive Orders“ und kann so jederzeit von einem neuen Präsidenten rückgängig gemacht werden.

44 Tom Hamburger / Peter Wallsten, a.a.O., S. 115.

45 Vgl. Pew Research Center for the People \& the Press, Faith-Based Funding Backed, a.a.O. 
ein menschliches Gesicht zu verleihen “46. Diese Vorgehensweise wurde für besonders effektiv gehalten, gilt doch "the minister [Priester] as the number one influencer in the African American community" 47 . Zwischen 2002 und 2004 wurden 15.000 religiöse Führer schwarzer, weißer und hispanischer Herkunft zu derartigen Veranstaltungen eingeladen. "They were hardly pep rallies for the President. But the conferences sent a resounding political message to all faith-oriented constituencies: President Bush cares about you." ${ }^{48}$ Ziel dieser Strategie war es, zumindest einen kleinen Teil des afroamerikanischen Wahlvolkes für die Republikaner zu gewinnen - und damit das politische Gleichgewicht in umstrittenen Staaten zu Gunsten der GOP zu beeinflussen. Unabhängig davon wurden Gelder des „Compassion Fund“ auch gezielt eingesetzt, um Kritiker zum Schweigen zu bringen. So erhielt die Organisation des evangelikalen TV-Predigers Pat Robertson nach heftiger Kritik an der Initiative des Präsidenten 1,5 Millionen US-Dollar. ${ }^{49}$

Der mitfühlende Konservatismus und die Faith Based Initiatives stellen also keinesfalls eine ideologisch verblendete Programmatik dar, sondern müssen als mehrdimensionale Strategie angesehen werden, die die persönliche Überzeugung des Präsidenten mit gesellschaftlichen Entwicklungen, parteipolitischen Notwendigkeiten und kühl kalkulierten wahltaktischen Zielsetzungen verband. Auch in Bezug auf die Agenda der Administration lamentierte John DiIulio, erster und bereits nach einem halben Jahr abgetretener Direktor des „OFBCI“, im Januar 2003: „What you've got is everything - and I mean everything being run by the political arm. ${ }^{50}$ Dabei spielen unterschiedliche Zielsetzungen eine Rolle, die allesamt zur Absicherung politischen Einflusses dienen sollten. Neben der Festigung der eigenen Stammwählerschaft (Sozial-Konservative) versprach sich Bushs Wahlkampfteam und später auch seine Administration eine Schwächung beziehungsweise innere Spaltung der Demokraten, ein verbessertes Ansehen bei Minderheiten sowie moderaten Wählern und einen daraus resultierenden, entscheidenden Vorteil in den battleground states.

\section{Gesellschaftliche Umgestaltung und "public policy"}

Die Präsidentschaft Bushs war hauptsächlich im Verlaufe der ersten Amtszeit von legislativen Erfolgen gekrönt. Begünstigt durch die Ereignisse des 11. Septembers 2001 und der darauf folgenden "rally around the flag" gelang es der Administration, in relativ kurzer Zeit maßgebliche Gesetzesinitiativen und damit wichtige Wahlkampfversprechen umzusetzen. Noch im Juni $2001 \mathrm{kam}$ es zu den massivsten Steuersenkungen seit der Präsidentschaft Reagans sowie im Jahre 2002 zu einer fast schon historischen Bildungsreform. Während diese beiden Initiativen von überparteilichen Mehrheiten getragen wurden, erlangte die stark umstrittene Reform des „Medicare-Entitlements“, des Krankenversicherungsanspruchs für Rentner, ihre legislative Geltung erst nach tumultartigen Abstimmungskämpfen

46 Thomas B. Edsall / Alan Cooperman, GOP Using Faith Initiative to Woo Voters, in: The Washington Post vom 15. September 2002, S. A5, eigene Übersetzung.

47 Matthew Dowd, Interview mit Peter Wallsten, in: Tom Hamburger / Peter Wallsten, a.a.O., S. 115.

48 David Kuo, a.a.O.

49 Vgl. Thomas B. Edsall, a.a.O., S. 82; vgl. Stanley W. Carlson-Thies, Implementing the faith-based Initiative, in: The Public Interest, Frühjahr 2004, S. 67.

50 Ron Suskind, Why are those men laughing, in: The Esquire Magazine, Januar 2003. 
scharf entlang der Parteigrenzen. Das zentrale Reformvorhaben der Bush-Administration, die Teilprivatisierung des Rentensystems, scheiterte im Jahre 2005 vollständig.

Obwohl die Administration und ihre Verbündeten im US-Kongress fast alle wesentlichen Wahlkampfversprechungen Realität werden ließen und damit eine nicht unerhebliche Dynamik unter Beweis stellten, wurden die Gesetze von konservativer wie auch liberaler Seite scharf kritisiert. Konservative Politiker und Intellektuelle bemängelten insbesondere den ideologischen Paradigmenwechsel, den die Bush-Administration mit ihrer kostenintensiven und aktivistischen Politik vollzogen habe. Den Demokraten indessen gingen die staatlichen Ausgaben nicht weit genug. Sie sahen in der Politik des republikanischen Präsidenten vermögende US-Bürger im Vorteil und bezeichneten die moderaten Strukturreformen als getarnte Taktiken, die auf eine Unterminierung der sozialvertraglich manifestierten Programme durch ,policy layering“ abzielten. ${ }^{51}$

Eine umfangreiche Analyse der benannten Gesetzgebungen ${ }^{52}$ gibt beiden Positionen Recht, wenn auch unter teils weitreichenden Einschränkungen. Einerseits geht die Politik der Bush-Administration mit stark ansteigenden Ausgaben einher, versetzt die USA in eine budgetpolitisch angespannte Lage, definiert die Rolle der Bundesregierung in unorthodoxer Weise neu und leistet so dem Zuweisungsföderalismus neuen Vorschub. Andererseits beinhalten die Initiativen Elemente, die eine langfristige Umgestaltung der in den USA lediglich rudimentär vorhandenen Sozialsysteme und eine Liberalisierung des Bildungswesens bezwecken. Dabei scheinen die Bedenken und Vorbehalte der Demokraten durchaus berechtigt, denn eine derartige Umgestaltung hat großes Potenzial, gesellschaftliche Veränderungen einzuleiten, die strukturell die konservative Sache begünstigen.

\subsection{Bildungspolitik}

Die Bildungsreform, der „No Child Left Behind Act“ (NCLB) kann zunächst als historisch bezeichnet werden, denn damit wurde die erste weitreichende Reform des Schulsystems auf Sekundarstufe seit dem „Elementary and Secondary Education Act“ aus dem Jahre 1965 realisiert. Vor dem Hintergrund des innenpolitisch dominierten Wahlkampfes des Jahres 2000 kam dem Bildungsthema eine wesentliche Rolle zu - auch deshalb, weil das Bildungssystem in den USA als weitgehend defizitär und mangelhaft wahrgenommen wurde. So bezeichneten 17 Prozent der Befragten Bildung als das wichtigste innenpolitische Thema, das auch die Liste der größten Herausforderungen für die USA anführte. ${ }^{53}$ Der Kandidat

51 Vgl. Jacob S. Hacker, Privatizing Risk without Privatizing the Welfare State: The Hidden Politics of Social Policy Retrenchment in the United States, in: American Political Science Review, 98. Jg. (2004), H. 2, S. 243 - 260. Auch in einer weiteren Publikation kritisierten Hacker und Pierson die Taktik klandestiner Reformen und Politiken und gingen dabei so weit zu sagen, dass dadurch der demokratische Prozess unterminiert werde: „The devil in the details of recent policies is not complexity, which is all but unavoidable in modern governance. It is policy features that are designed to hide what policies are really doing while deliberately restricting the scope for future democratic choice." Jacob S. Hacker / Paul Pierson, Off Center, The Republican Revolution and the Erosion of American Democracy, New Haven 2006, S. 17.

52 Der Autor arbeitet an einem Promotionsprojekt zum Thema Gesundheits- und Sozialpolitik.

53 Vgl. Jeffrey M. Jones, Slim Majority Dissatisfied with Education in the U.S., Gallup Poll News Service, 8. September 2005. 
Bush machte Bildungsfragen zum zentralen Gegenstand seines Wahlkampfes und trug so den gesellschaftlichen Erwartungen Rechnung.

Sowohl die Demokraten als auch die Republikaner sind seit den 1960er Jahren darum bemüht, sich jeweils als die Partei darzustellen, welche die Struktur und das Fundament der US-Gesellschaft als „opportunity society“, als Gesellschaft der Chancen verteidigt. Bildung stellt in diesem Kontext eine entscheidende Komponente dar, da der gleichberechtigte $\mathrm{Zu}$ gang zu ihr als Ausgangspunkt gesellschaftlicher Chancengleichheit gesehen werden kann. Doch traditionell wird der Demokratischen Partei eine höhere Kompetenz in bildungsbezogenen Themen zugesprochen. Daher ist die Herangehensweise des Bush-Teams, im Wahlkampf 2000 Bildung zu einer Top-Priorität zu machen, als logischer und politisch kluger Schachzug zu werten. Jedwede Forderung nach einer Drosselung der Bildungsausgaben oder gar einer Abschaffung des Bildungsministeriums wäre politisch überaus unklug gewesen: „Education was certainly very high in what the public was concerned about in the late nineties through about 2001. Just certainly Bush was working in an environment where he wanted to get education addressed because that was a real problem for the Republicans." 54

Während 1996 Präsident Clinton klar die führende Kompetenz in Bildungsfragen verkörperte, gelang es Bush, den Wechsel von der Anti-Bildungspartei hin zur in der Wählerperzeption gleich starken Kompetenz in den Jahren 2000 und 2004 zu vollziehen und damit wichtige Punkte in der Wählergunst der amerikanischen Mittelschicht und Arbeiterklasse zu sammeln (vgl. Tabelle 3). Diese repräsentierten einerseits stets eine Domäne der Demokraten; andererseits sind Bildungsfragen sowie Bildungsqualität zentrale Themen für sie. ${ }^{55}$ Der Kandidat Bush verfolgte dabei eine Strategie, die es den Demokraten nahezu unmöglich machte, effektiv zu reagieren. ${ }^{56}$ Besonders die Abkehr von der Ansicht, die Bundesregierung habe eine untergeordnete, minimale oder gar keine Rolle in Bildungsfragen zu spielen, zahlte sich für die Republikaner aus.

Bush gelang es also, den traditionellen Vorteil der Demokraten in Bildungsfragen aufzulösen. Man traute es dem Kandidaten Bush mehr zu, mit einer Agenda der Liberalisierung einerseits und einem strikten Kontrollregime andererseits die strukturellen Defizite im Bildungswesen zu beseitigen und damit die Chancengleichheit zu erhöhen. Dabei spielt auch die Zuweisung von Mitteln eine Rolle. Traditionell ist die Finanzierung der Schulen auf lokaler Ebene verwurzelt. Gelder fließen analog zu den Einnahmen des jeweiligen Distrikts aus der Haus- und Grundsteuer. So stehen „reichen“ Gegenden automatisch mehr Mittel für das Schulwesen zur Verfügung, armen Gegenden entsprechend weniger. Der „No Child Left Behind Act“ stellt nun neue Gelder zur Verfügung, bindet diese jedoch an ein standardisiertes Testverfahren und definiert Bildungsstandards, die regelmäßig überprüft beziehungsweise realisiert werden müssen. Insgesamt stiegen die in diesem Zusammenhang ein-

54 Eigenes Interview mit Rick Hess, American Enterprise Institute, Washington DC, 17. September 2006.

55 Vgl. PSRA / Newsweek Poll, Oktober 2000, und Princeton Survey Research Associates International / Newsweek Poll, Oktober 2004, Zugriff unter: www.ropercenter.uconn.edu/iPOLL.html (Zugriff am 19. September 2006).

56 „In many ways he stole their ideas and their language and their record. There was not much of an opportunity for Al Gore to go to the left on Education." Eigenes Interview mit Mike Petrilli, Fordham Foundation, Washington DC, 3. Oktober 2006. Siehe ebenso Andrei Cherny, Pro Choice, A Democratic Ownership Society, in: The New Republic vom 1. November 2004, S. 24. 


\begin{tabular}{|l|l|l|}
\hline \multicolumn{3}{|c|}{ Tabelle 3: $\begin{array}{l}\text { Kompetenz der US-Präsidentschaftskandidaten in Bildungsfragen in der } \\
\text { Wählerperzeption, 1984 bis 2004 (in Prozent) }\end{array}$} \\
\hline Jahr & Demokrat & Republikaner \\
\hline 1984 & 39 (Walter Mondale) & 43 (Ronald Reagan) \\
\hline 1988 & 51 (Michael Dukakis) & 34 (George Bush sen.) \\
\hline 1992 & 55 (Bill Clinton) & 35 (George Bush sen.) \\
\hline 1996 & 57 (Bill Clinton) & 27 (Bob Dole) \\
\hline 2000 & 46 (Al Gore) & 45 (George W. Bush) \\
\hline 2004 & 47 (John Kerry) & 46 (George W. Bush) \\
\hline $\begin{array}{l}\text { Quelle: Frederick M. Hess / Patrick McGuinn, Seeking the Mantle of Opportunity: Presidential Politics } \\
\text { and the Educational Metaphor, in: Frederick M. Hess (Hrs.), Tough Love for Schools, Essays on Com- } \\
\text { petition, Accountability, and Excellence, Washington DC 2006, S. 29. }\end{array}$ \\
\hline
\end{tabular}

schlägigen Ausgaben unter „Title I“ des „Elementary and Secondary Education Act“ von 2001 bis 2007 um 45 Prozent. $^{57}$

Nicht nur die neue Ausgabenpolitik der Bush-Administration kam den Schulen zugute. Auch strukturelle Veränderungen, allen voran die Einführung von Marktmechanismen und neuartige Elemente privater Bildung, sprachen nicht nur republikanische Basiswähler an. Zwar wurde im Zuge der parlamentarischen Verhandlungen auf die Einführung eines reinen Gutscheinsystems verzichtet, jedoch steht es Eltern und Schülern offen, unter bestimmten Voraussetzungen einen Schulwechsel zu vollziehen. Im Rahmen dieses Ansatzes, der unter dem Oberbegriff „Public School Choice“ zusammengefasst wird (in klarer Abgrenzung zu „Private Choice“), können die Betroffenen innerhalb des Schulbezirks (innerdistrict) oder über dessen Grenzen hinaus (interdistrict) eine andere öffentliche Schule wählen. Zudem besteht die Möglichkeit eines Wechsels zu "Charter Schools“, die jedoch nicht in allen Bundesstaaten verfügbar sind (gegenwärtig in 40 sowie in Puerto Rico und Washington DC). Die anfallenden Kosten müssen von den Einzelstaaten getragen werden. ${ }^{58}$

Charter Schools stehen nach wie vor unter staatlicher Aufsicht, werden jedoch von privaten Organisationen geleitet. Dieser Schultypus ist von vielen staatlichen Regularien befreit und hat daher eine größere Entscheidungsfreiheit bei Inhalten und Stundenplänen. Zuvor festgelegte Ziele müssen dabei aber stets erfüllt werden. Die Wahl der Schule ist in einigen Bundesstaaten bereits seit vielen Jahren möglich; das NCLB-Gesetz weitet dies auf alle Staaten aus und löst die Wahlfreiheit „automatisch“ bei schwachen Schülerleistungen aus. ${ }^{59}$ Die Gesetzgebung fördert den Aufbau von Charter Schools mit erheblichen finanziellen Mitteln und weist den Staaten zu diesem Zwecke block grants zu, die eine weitgehen-

57 Vgl. Department of Education, Summary of Discretionary Funds, Fiscal Years 2001 - 2007, 2. August 2006, Zugriff unter: www.ed.gov/about/overview/budget/budget07/07bylevel.pdf (Zugriff am 27. September 2006).

58 Die Staaten müssen grundsätzlich zwanzig Prozent der „Title I“-Gelder für potenzielle Transportkosten beiseitelegen, die aus den „School Choice“-Mechanismen entstehen können. Vgl. Frederick M. Hess / Michael J. Petrilli, No Child Left Behind Primer, New York 2006, S. 51.

59 Vgl. Richard Lee Colvin, Public School Choice: An Overview, in: Frederick M. Hess / Chester E. Finn Jr. (Hrsg.), Leaving No Child Behind? Options for Kids in Failing Schools, New York 2004, S. 11 - 19. Charter Schools existieren seit dem Jahre 1992 und werden seither immer mehr angenommen. 
de Autonomie bei der Anwendung der Gelder erlauben. ${ }^{60}$ Marktwirtschaftliche Mechanismen werden auch mit dem „Supplemental Educational Services“-Programm (SES) begünstigt, das ebenfalls nach fortgesetzt schwachen Leistungen ausgelöst wird und „einem Marktplatz für staatlich finanzierte Tutorien" ${ }^{61}$ gleichkommt.

Die Einführung privater Wahlmöglichkeiten durch Bildungsgutscheine findet großen Anklang nicht nur bei den republikanischen Basiswählern, die dies besonders aus ökonomischen und religiösen Gründen unterstützen, sondern auch verstärkt bei Minderheiten. In diesem Zusammenhang wird das große Potenzial des „opportunity“-Paradigmas deutlich, denn afroamerikanische und hispanische US-Bürger fühlen sich durch die oben skizzierte Finanzverfassung der Schulbezirke ihrer Möglichkeiten beraubt. Wie Statistiken des Bildungsministeriums belegen, liegen schwarze und auch hispanische Schüler bei den erfolgreichen Abschlüssen der Sekundarstufe (High School) in allen Staaten weit hinter ihren Mitschülern weißer Hautfarbe zurück. ${ }^{62}$ Dieses als „achievement gap“ bezeichnete Faktum wird indessen als gesamtgesellschaftliches Problem wahrgenommen. Im Jahre 2001, kurz vor der Verabschiedung des NCLB-Gesetzes, gaben 66 Prozent der US-Bürger an, die Überwindung der Leistungsschere sei außerordentlich wichtig. ${ }^{63}$

So verwundert es kaum, dass sich Afroamerikaner und Hispanier klar für Bildungsgutscheine aussprechen. Im Vorfeld der Präsidentschaftswahl im Jahre 2000 befürworteten 60 Prozent der Afroamerikaner und 70 Prozent der Hispanier die Einführung von Bildungsgutscheinen. ${ }^{64}$ Die von der Bush-Kampagne angeschlagene Rhetorik von „exit vouchers“ (als eine Art Freifahrtschein aus dem Ghetto) sowie von „opportunity scholarships“ ist nicht nur Teil des politisch-ideologischen Grundkonzepts des mitfühlenden Konservatismus ${ }^{65}$, sondern soll auch das Eintreten der Republikanischen Partei für die Erreichung gleicher Bildungschancen für alle symbolisieren und gleichzeitig den Demokraten, die traditionell den größeren Rückhalt bei dieser Wählergruppe genießen, in Bildungsfragen den Rang ablaufen. Mit dieser Rhetorik und der umfassenden Wahlkampfprogrammatik gelang es den Republikanern, das Ansehen der Partei bei den Minderheiten aufzubessern und darüber hinaus einen Keil in ein fundamentales Unterstützernetzwerk der Demokraten zu trei-

60 Vgl. David P. Smole, School Choice: Current Legislation, CRS Report for Congress, No. IB98035, 5. Mai 2006, S. 7.

61 Siobhan Gorman, The Invisible Hand of NCLB, in: Frederick M. Hess / Chester E. Finn Jr., a.a.O., S. 41. Das Volumen des Marktes privater Tutorien wird auf zwei Milliarden US-Dollar geschätzt.

62 Vgl. National Center for Education Statistics, The Condition of Education 2005, Indicator 24, High School Exit Examinations, Washington, 2005, S. 166, Zugriff unter: http://nces.ed.gov/ programs/coe/2005/pdf/24_2005.pdf (Zugriff am 21. September 2006). Am weitesten klafft die Lücke bei den erfolgreichen Abschlüssen in Arizona und Indiana auseinander. Dort liegen weiße Schüler mit mehr als 35 Prozent vor den schwarzen und hispanischen Schülern.

63 Vgl. Julie Ray, Public: Parents Pivotal to Student Performance, The Gallup Poll Tuesday Briefing, 27. September 2005.

64 Vgl. Pew Research Center for the People \& the Press, Issues and Continuity Now Working for Gore, 14. September 2000, Zugriff unter: http://people-press.org/reports/print.php3?ReportID $=33$ (Zugriff am 28. November 2006).

65 George W. Bush unterstrich selbst nochmals die zentrale Rolle der Bildungsreform im Konzept des compassionate conservatism: „Finally, to close the gap of hope, America must make a solemn commitment: Every child will be educated and no child will be left behind." Ders., A Charge to Keep, a.a.O., S. 232. 
ben. Während Afroamerikaner und Hispanier ein Gutscheinsystem befürworten, ist mit den Lehrergewerkschaften eine finanzkräftige Lobby mit hohem Ansehen insbesondere auf lokaler Ebene strikt gegen einen solchen Schritt. „Inner-city blacks are the number one supporter [of vouchers], they hate their schools and they think they benefit from vouchers and in all the public opinion polls blacks support vouchers. But black politicians in Congress, African-American, Urban Democrats are all against vouchers. And they are completely out of touch with their constituents." 66

Die Bildungspolitik der Republikaner, die sich die Beendigung der „charakterlosen Diskriminierung in Bildungsfragen "67 auf die Fahnen schrieben, konnte das Bild des mitfühlenden Konservatismus effektiv kommunizieren. Zwar haben sich die Afroamerikaner weder in den Wahlen 2000 noch 2004 in Massen zur Republikanischen Partei bewegt, dennoch kann der politische Nutzen vor allem im Rahmen der Wahlkämpfe als groß angesehen werden, da die Wahlen sehr knapp ausgingen.

\subsection{Steuern, Eigentum und die sozialen Sicherungssysteme}

Obgleich die sozialen Sicherungssysteme in den USA aus europäischer Sicht keine nennenswerte Ausprägung aufweisen, haben sich die sozialen Errungenschaften des „New Deal“ und der „Great Society“ als wesentlicher Bestandteil im US-amerikanischen Sozialvertragsdenken etabliert und genießen gesellschaftlich hohes Ansehen. Jedwede Reformmaßnahme, die nur im Entferntesten an den Grundfesten von „social security“ (Rente) und „Medicare“ (Krankenversicherung für Rentner) rüttelt, gilt als politisch riskant - der beide Programme untermauernde Sozialpakt („social compact“) wird daher als „third rail of politics" bezeichnet. Eine Reform dieser Grundsicherung unterliegt zudem einer ausgeprägten Pfadabhängigkeit, die sich in einer Vielzahl gesellschaftlicher und wirtschaftlicher Interessen manifestiert. ${ }^{68}$

Unabhängig davon setzte sich der Kandidat Bush im Wahlkampf 2000 für eine Reform beider Systeme ein. Kernstück dieser Vorhaben war die Schaffung einer „ownership society“, die in der Theorie die Wahlfreiheit des Einzelnen steigern und gleichzeitig jeden Leistungsempfänger zum alleinigen „Eigentümer“ seiner individuell und eigenverantwortlich angesparten Sozialleistungen machen sollte. „I think part of government's responsibility is

66 Eigenes Interview mit Thomas Loveless, 22. September 2006; vgl. ebenso eigenes Interview mit Mike Petrilli, 3. Oktober 2006: „The [Democratic] candidates are paying more attention to the teacher union's leadership who are opposed to vouchers and paying less attention to its grass roots. I think that represents some opportunities for Republicans for the choice movement." Besonders schwer wiegt diese Tatsache, da sich auch die mächtigen und finanzstarken Lehrerverbände gegen Vouchers wenden - die demokratischen Abgeordneten und Kandidaten jedoch maßgeblich auf deren Unterstützung angewiesen sind: „... the White House sought to drive a wedge between two core Democratic Constituencies: the teachers unions ... and the African American and Latino parents..." Tom Hamburger / Peter Wallsten, a.a.O., S. 97.

67 George W. Bush, Acceptance Speech Republican National Convention, Philadelphia, 3. August 2000, eigene Übersetzung. Der Originalwortlaut lautete, „to end the soft bigotry in education”.

68 Zur Pfadabhängigkeit und Reform der sozialen Sicherungssysteme siehe Paul Pierson, Increasing Returns, Path Dependence, and the Study of Politics, in: American Political Science Review, 94. Jg. (2000), H. 2, S. 251-267. 
to encourage certain cultures. And a primary cultural change that I have been trying to instill [...] is a new period of personal responsibility." ${ }^{69}$ Im Geiste des britischen Premierministers Benjamin Disraeli, der anmerkte, die Konservativen hätten zukünftig die besten politischen Chancen, wenn es gelänge, eine „property-owning democracy“ zu schaffen, zielt auch die „ownership society“ auf langfristige Dominanz ab.

Die Vision dahinter hat eine doppelte Bedeutung: Einerseits könnte so eine endgültige Erosion der „New Deal“-Koalition bewirkt und der nach wie vor präsente politische Griff der Demokraten um die Sozialprogramme gelockert werden. Plötzlich würden die Republikaner als jene Partei wahrgenommen werden, die den Sozialpakt modernisiert und die finanziell bedrohten „entitlement“-Programme in das nächste Jahrhundert geführt haben. Andererseits zieht die strukturelle Funktionsweise der „ownership society“ eine fast logische Bindung an die Republikaner nach sich. Im Plan der Bush-Strategen ist „ownership“ mit Aktienbesitz gleichzusetzen beziehungsweise hängt stark mit der Besteuerung von angespartem Wohlstand zusammen: Jeder Amerikaner soll so zu einem kleinen Investor werden. „More investors means more people with a stake in corporate America who will be sceptical about business bashing .... [like] Al Gore's „the people vs. the powerful” campaign in 2000."70 Auf diesem Wege - soweit die Theorie - könne „the Ownership Society ... the ownership realignment" 71 werden.

In der Praxis erwies sich dieser Plan als kaum durchsetzbar. Das Kernstück der „Eigentümergesellschaft", die Reform des Rentensystems, blieb trotz intensivster Lobbyarbeit und einer nationalen, persönlich vom Präsidenten durchgeführten Werbestrategie erfolglos. „Personal Retirement Accounts“ (PRAs) werden zumindest nicht mehr mit George W. Bush als Präsident eingeführt werden. Dabei war allerdings nicht die Teilprivatisierung an sich, die von mehr als zwei Dritteln der jüngeren Wähler begrüßt worden wäre ${ }^{72}$, der Grund für das Scheitern. Vielmehr ist in der 2005 angespannten Budgetsituation der Hauptgrund zu suchen. Daher wandten sich auch fiskalisch konservative Republikaner, die nicht willens waren, die enormen Übergangskosten der Privatisierung mit zu verantworten ${ }^{73}$, gegen den Plan der Administration und trugen so entscheidend dazu bei, dass die Initiative rasch von der Tagesordnung verschwand.

In anderen Bereichen war die Bush-Administration aber sehr wohl in der Lage, ihre Politik umzusetzen, beispielsweise in der Steuergesetzgebung aus den Jahren 2001 und 2003. In der Tradition der angebotsorientierten Politik richtete sich der „Economic Growth and Tax Relief Reconciliation Act“ (EGTRRA) an der Reduzierung der Grenzsteuersätze aus. ${ }^{74}$ Diese wurde 2001 in allen Steuerkörben umgesetzt. Zudem gibt der EGTRRA wesentliche neue Anreize für die private Altersvorsorge. Die Beitragsobergrenzen zu „Individual Retirement Accounts“ (IRA) werden bis zum Jahre 2008 von $\$ 2.000$ jährlich auf $\$ 5.000$ anstei-

69 George W. Bush, zitiert in: Fred Barnes, a.a.O. (2006), S. 128.

70 John Micklethwait / Adrian Woolridge, a.a.O., S. 245.

71 Greg Strimple, zitiert in: Tom Hamburger / Peter Wallsten, a.a.O., S. 199. Strimple ist ein republikanischer Meinungsforscher.

72 Vgl. John Micklethwait / Adrian Woolridge, a.a.O., S. 245.

73 Vgl. Ron Suskind, The Price of Loyality, London 2004, S. 194.

74 Vgl. Joachim Nagel, Supply-Side Policy in den USA. Eine theoretische und empirische Analyse der angebotsorientierten Wirtschaftspolitik Reagans unter Berücksichtigung besonderer steuerlicher Aspekte, Berlin 1997. 
gen und danach an die Inflation gekoppelt. ${ }^{75}$ Neben der Erhöhung der privaten Sparquote erhoffte sich die Administration dadurch eine verstärkte Partizipation an privaten Rentenplänen. In der Tat sind IRAs wichtige Säulen der Altersvorsorge geworden. Im Jahre 2003 sparten bereits 33,3 Prozent der US-amerikanischen Haushalte in solchen Anlagen Kapital für das Alter an. ${ }^{76}$ Hinzu kommt eine Vielzahl von Änderungen bezüglich betrieblicher Vorsorgemöglichkeiten über so genannte 401(k)-Sparpläne. Nach dem EGTRRA können ab dem Jahre 2006 \$15.000 jährlich in diese Art der Vorsorge einbezahlt werden, doppelt so viel wie zuvor. Insgesamt wurden die Vorsorgemöglichkeiten über private und betriebliche Rentenpläne weitgehend reformiert und erheblich ausgeweitet. Durch vereinfachte Regelungen und höhere Beitragsmöglichkeiten wurden auch Anreize für kleine Unternehmen geschaffen, einen betrieblichen Rentenplan aufzustellen. Der Ausbau privater Vorsorgemöglichkeiten und Vermögensbildung wird durch die Erhöhung der Beitragsobergrenzen zu von der Steuer befreiten „Education IRAs“ von \$500 auf jährlich $\$ 2.000$ abgerundet. $^{77}$ Außerdem wird die Erbschaftssteuer jährlich reduziert, bis sie im Jahre 2010 völlig aufgehoben wird, und die Kapitalertragssteuer wird gesenkt. Nach dem „Jobs Growth and Tax Relief Reconciliation Act" (JGTRRA) des Jahres 2003, der nur durch die entscheidende Stimme des Vizepräsidenten Dick Cheney zustande kam, müssen Geringverdiener ab dem Jahre 2008 keine Steuern mehr auf Dividenden und Kapitalerträge abführen (die übrigen Einkommensklassen 15 Prozent). ${ }^{78}$

Abschließend sei auf den „Prescription Drug and Medicare Modernization Act“ (MMA) hingewiesen. Die Gesetzgebung, die das Medicare-Programm um eine freiwillige Medikamentenversicherung ergänzt und damit erstmals seit der Präsidentschaft Johnsons einen neuen Anspruch schuf (wohlgemerkt unter dem Republikaner George W. Bush), ging mit weiteren Privatisierungstendenzen einher, von denen hier lediglich die für „ownership“ relevante Strukturreform erwähnt werden soll. „Health Savings Accounts“ (HSAs) wurden selbst von Newt Gingrich, ungeachtet der massiven Kritik an der Gesamtgesetzgebung, als Argument ins Feld geführt, für den MMA zu stimmen. ${ }^{79}$

Allerdings sind HSAs außerhalb des Medicare-Entitlements anzusiedeln und wurden lediglich im Rahmen der Gesetzgebung autorisiert. Dabei handelt es sich um Versicherungspolicen, die an eine hohe Selbstbeteiligung gekoppelt sind und gleichzeitig steuerfreie Sparkonten ermöglichen. Eine derartige Versicherung muss eine jährliche Selbstbeteiligung von mindestens $\$ 1.000$ beinhalten. Dazu kann der Versicherungsnehmer jährlich einen

75 Vgl. David L. Brumbaugh / Jane G. Gravelle / Steven Maguire / Louis Alan Talley u.a., 2001 Tax Cut: Description, Analysis, and Background, CRS Report for Congress, 9. Dezember 2002.

76 Vgl. Investment Company Institute, Mutual Fund Fact Book 2004, S. 88, Zugriff unter: http:// www.ici.org/stats/latest/2004_factbook.pdf (Zugriff am 16. April 2005). Zum Vergleich: In Deutschland sparen 14,8 Prozent über das vergleichbare Riestermodell. Vgl. Kerstin Windhövel, Kapitalakkumulation durch die Riester-Rente, Friedrich-Alexander-Universität Erlangen-Nürnberg, 2004, S. 1, Zugriff unter: http://doku.iab.de/grauepap/2004/Windhoevel_Riester-Rente. pdf (Zugriff am 17. April 2005).

77 Vgl. David L. Brumbaugh / Jane G. Gravelle / Steven Maguire / Louis Alan Talley u.a., a.a.O., S. 10.

78 Vgl. Joint Committee on Taxation, Summary of the Conference Agreement on H.R.2. Jobs Growth and Tax Relief Reconciliation Act, Washington DC, 22. Mai 2003, Zugriff unter: http:// www.house.gov/jct/x-54-03.pdf (Zugriff am 3. November 2005).

79 Vgl. Newt Gingrich, Conservatives Should Vote "Yes" on Medicare, in: The Wall Street Journal vom 20. November 2003. 
Betrag von maximal \$2.600 in einen HSA überweisen. Die Beitragszahlungen sind steuerfrei, werden also vor der Ermittlung der Steuerschuld vom Brutto-Einkommen abgezogen. Darüber hinaus erfolgt eine steuerfreie Auszahlung des Ersparten, wenn dieses zur Deckung von Gesundheitsausgaben verwendet wird. ${ }^{80} \mathrm{HSAs}$ stellen eine neue Option der Krankenversicherung dar, sind jedoch keine neue Idee. Bereits in den 1970er Jahren wurden entsprechende Pläne ausgearbeitet, scheiterten jedoch immer wieder im Kongress. Erst 1996 wurde das Konzept in Form von „Medical Savings Accounts“ verabschiedet, aber nur stark limitiert umgesetzt. ${ }^{81}$ Nach deren Einführung im Jahre 2004 haben sich bisher vergleichsweise wenig Bürger für HSAs entschieden; die Policen verzeichnen aber einen starken $\mathrm{Zu}$ wachs: So hat sich die Zahl der HSA-Eigner im Januar 2006 im Vergleich zu 2005 verdreifacht - 3,2 Millionen Personen verfügen nun über HSAs. ${ }^{82}$

Vertreter von US-Think Tanks bezeichneten in Interviews HSAs als „ideological weapon" 83 , andernorts als „major breakthrough" 84 und schließlich wegen ihrer beschränkten Reichweite und vor dem Hintergrund der in konservativen Kreisen ungeliebten Gesamtgesetzgebung als „miss set of priorities“ ${ }^{85}$. Festzuhalten bleibt, dass sie einen wesentlichen Beitrag zur „ownership society“ leisten und damit maßgeblich, wenn auch nur mittelfristig, zu einer gesellschaftlichen Umgestaltung im Sinne konservativ-libertärer Politik führen.

\section{Die Professionalisierung der Republikanischen Wahlkampfführung}

Das politische System der USA ist von einer raschen Abfolge von Wahlen bestimmt - eine Konzeption, die in der Intention der Gründungsväter ein Teilelement im Gewaltenteilungssystem von checks and balances darstellt. Diese hohe Frequenz von Wahlen, insbesondere zum Repräsentantenhaus, dessen Abgeordnete sich alle zwei Jahre zur Wiederwahl stellen müssen, und der damit verbundene Kampf um die Wählergunst haben den Begriff der „permanent campaign“ geprägt. So trifft für viele Abgeordnete, aber auch in zunehmenden Maße für den Präsidenten die etwas platte Floskel „vor der Wahl ist nach der Wahl“ zu. Der „permanente (Wahl-)Kampf“ der Mandatsträger wirkt sich vielfältig auf die politischen Entscheidungsstrukturen aus. Außerdem zieht er einen hohen Bedarf an finanziellen Mitteln, einen gesicherten und strukturierten Zugang zur Presse sowie ein möglichst detailliertes Wissen um Wähler und Wählerstrukturen auf lokaler wie nationaler Ebene nach sich.

80 Vgl. Bob Lyke / Chris Petersen, Health Savings Accounts, CRS Report for Congress, 24. September 2004. Die Beitragszahlungen sind an die jährliche Inflationsrate gekoppelt und steigen daher sukzessive an.

81 Vgl. Victoria Craig Bunce, Medical Savings Accounts, Progress and Problems under HIPAA, Cato Policy Analysis, No. 411, 8. August 2001, S. 8 ff.

82 Vgl. Jeff Lemieux, January 2006 Census Shows 3.2 Million People Covered by HAS Plans, Center for Policy and Research, 2006, Zugriff unter: http://www.ahip.org/content/default. aspx?docid=15302 (Zugriff am 25. November 2006). Laut der Studie waren 31 Prozent der HSAEigner zuvor ohne jeden Versicherungsschutz.

83 Eigenes Interview mit Len Nicols, The New America Foundation, 10. November 2006.

84 Eigenes Interview mit Joe Antos, American Enterprise Institute, 7. November 2006.

85 Eigenes Interview mit Bob Moffit, Heritage Foundation, 14. November 2006. 
In mindestens zwei der vorgenannten Bereiche konnte sich die Republikanische Partei einen wesentlichen strukturellen Vorteil erarbeiten. Regelmäßig gelingt es ihr, die Demokraten in punkto Spenden und Wahlkampffinanzierung zu übertreffen. Von zentraler Bedeutung ist dabei das „K Street Project“. Benannt nach einer Straße, die mitten durch Downtown Washington DC führt und Heimstatt zahlreicher Unternehmen, Lobbyisten und Think Tanks ist, schuf das Projekt eine effektiv und straff organisierte Symbiose von Lobbyisten, Großindustrie und der Republikanischen Partei. Neben dem Anti-Steuer-Aktivisten und Gründer des Projekts Grover Norquist verstanden es vor allem Newt Gingrich und Tom DeLay, dieses 1995 gegründete Netzwerk zu spinnen und weiter auszubauen. Doch erst unter der Präsidentschaft George W. Bushs entfaltete es seine ganze Wirkung. ${ }^{86}$ Erklärtes Ziel und bald gängige Praxis der Parteistrategen um Senator Rick Santorum (Pennsylvania) war es, eine möglichst große Anzahl von Parteifreunden oder vorherigen Mandatsträgern in vakante Positionen der Interessengruppen zu befördern und damit den Kurs der Lobbyisten entscheidend mitzusteuern. „Lobbying organizations got the message: if they wanted to have good relations, or even get appointments to see the leaders of Congress, they needed to have Republicans in top positions." 87

Zwischen 2000 und 2003 wechselten 17 frühere Kongressmitglieder die Seite und übernahmen Posten an der K Street. Nur zwei davon gehörten den Demokraten an. ${ }^{88} \mathrm{Wie}$ die liberale Zeitung „New Republic“ anklagend und zugleich frustriert bemerkte, waren die Republikaner so imstande, neben der personellen Durchdringung der Interessengruppen sich auch „a self-perpetuating money machine” 89 zu besorgen.

Auf diese Weise verschafften sie sich nicht nur einen finanziellen Vorteil - 2002 erhielten sie 63 Prozent aller von Lobbyisten ausgeschütteten Wahlkampfgelder („soft money“). ${ }^{90}$ Auch die Unterstützung durch die Unternehmen und Interessengruppen im Wahlkampf zahlte sich aus. Da Zuwendungen von „Political Action Committees“ (PACs) gesetzlichen Regularien unterliegen und limitiert sind, haben Großunternehmen und Handelsvereinigungen ihre Aktivitäten mit „soft money“ signifikant gesteigert und engagieren sich gezielt in einzelnen Wahlkreisen. ${ }^{91}$ Dabei ergreifen mit den Republikanern verbundene Unternehmen direkt Partei für spezifische Gesetzesvorhaben; beispielsweise sandte General Motors an all seine Aktienteilhaber eine Broschüre, in der sich das Unternehmen klar für die Senkung der Dividendenbesteuerung aussprach, und die Investmentbank Morgan Stanley verschickte zusammen mit ihrem Jahresbericht einen Brief des Vorstandsvorsitzenden gleichen Inhalts an ihre Kunden. ${ }^{92} \mathrm{Zu}$ ähnlichen Aktionen kam es im Zuge der Medicare-Gesetzge-

86 Vgl. Jim VandeHei / Juliet Eilperin, Targeting Lobbyists Pays Off for GOP, in: The Washington Post vom 26. Juni 2003, S. A1.

87 Tom Hamburger / Peter Wallsten, a.a.O., S. 178.

88 Vgl. Gail Russell Chaddock, Republicans take over K Street, The Christian Science Monitor, 29. August 2003, S. 1. Bei Erscheinen des Artikels waren insgesamt 160 frühere Kongressmitglieder als Lobbyisten beschäftigt.

89 Jonathan Chait, a.a.O., S. 22.

90 Vgl. Center for Responsive Politics, Soft Money for Lawyers \& Lobbyists in 2002, Zugriff unter: http://www.opensecrets.org/softmoney/softindus.asp?ind=K (Zugriff am 30. August 2007).

91 Zahlen finden sich auch auf der Website des Center for Responsive Politics, Soft Money Donor Search, Zugriff unter: http://www.opensecrets.org/softmoney/index.asp (Zugriff am 30. August 2007). Vgl. Nicholas Confessore, Welcome to the Machine, How the GOP disciplined K Street and made Bush Supreme, in: The Washington Monthly, Juli/August 2003.

92 Ebenda. 
bung, als sich die Pharmaindustrie massiv für den Vorschlag der Bush-Administration einsetzte.

Die Republikanische Partei, die im Geiste der „Republican Revolution“ der Korruption auf Capitol Hill ein Ende bereiten und sich als „honest broker“, frei von Partikularinteressen, ausschließlich für das Wohl des Wählers einsetzten wollte, wurde nun zum größten Nutznießer der finanziellen Zuwendungen der Großunternehmen und verstrickte sich in

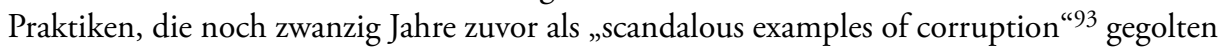
hätten.

Neben der engen Verflechtung der Partei mit Lobbyisten und Interessengruppen entwickelte die GOP unter der Federführung von Karl Rove ein ausgereiftes politisches Marketingsystem, das gezielt in den Wahlkämpfen eingesetzt wurde. „Voter Vault“, ein Datensatz, der vom Zeitungsabonnement über Essgewohnheiten bis hin zum Fitnessclub alle möglichen Informationen enthält, umfasst mittlerweile 176 Millionen Bürger und 111 Millionen Haushalte. ${ }^{94}$ Damit wird nicht nur ein zielgerichtetes politisches Direkt-Marketing möglich, sondern es können auch potenzielle Wähler aufgespürt werden, die von traditionellen Kampagnen übersehen würden. ${ }^{95}$

Ziel dieser Vorgehensweise ist es, möglichst viele Menschen noch in letzter Sekunde an die Wahlurnen zu bringen und sich so in engen Wahlkämpfen den entscheidenden Vorteil zu sichern: „In 2002, for the first time in recent memory, Republicans ran better get-outthe-vote programs than Democrats. When well done, such drives typically raise a candidate's Election Day performance by two to four percentage points." 96 Eine höhere Wahlbeteiligung wird auch erzielt, weil mit Hilfe von Voter Vault insbesondere "single issue voters“ identifiziert und gezielt umworben werden können. Dieser Wählertypus orientiert sich stark an einzelnen Themen und gilt deshalb auch als Wechselwähler. Durch die Anwendung der Datensätze konnten die Republikaner zuvor als homogen perzipierte Wählerblöcke segmentieren. So konnte eine große Anzahl von Personen mit maßgeschneiderten Themen angesprochen werden, etwa politisch konservative und pro Israel orientierte Juden, sozial konservative Schwarze oder Arbeitnehmer mit geringem Einkommen. Diese Gruppen werden mit Informationen versorgt, die bewusst auf Reizthemen - zum Beispiel Kampf gegen Terrorismus, Homo-Ehe, Abtreibung, Einwanderung oder Steuersenkungen - zugespitzt sind. Im Wahlkampf 2004 wurden zudem in 20 Schlüsselstaaten Fragebögen an bis zu 12.000 Personen versandt, auf denen diese ihre persönlichen Reizthemen vermerken konnten.

Auf diese Weise die Beteiligung zu erhöhen war 2004 von entscheidender Bedeutung, denn der Anteil der als unabhängig eingestuften Wähler fiel zwischen 1988 und 2002 von 15 Prozent auf nur sieben Prozent. Beide Parteien bemühten sich daher, möglichst viele Wähler an die Urnen zu locken - und beide Parteien waren erfolgreich. Während im Jahre 2000105 Millionen US-Bürger wählten, waren es 2004122 Millionen. John Kerry gewann 16 Prozent mehr Stimmen als Al Gore (59 Millionen); Präsident Bush steigerte seinen Anteil um 23 Prozent auf 62 Millionen Stimmen. ${ }^{97}$

93 Thomas B. Edsall, a.a.O., S. 127.

94 Vgl. ebenda, S. 76.

95 Vgl. Tom Hamburger / Peter Wallsten, a.a.O., S. 151.

96 Thomas B. Edsall, Democrats' Data Mining Stirs an Intraparty Battle, in: The Washington Post vom 8. März 2006, S. A1.

97 Vgl. Michael Barone, a.a.O., S. 21 f. 
Die Datenbank Voter Vault sowie die damit verbundene Reizthemen-Strategie entpuppten sich als schlagkräftige Wahlkampfinstrumente. An ihrem Ausbau wird fieberhaft gearbeitet, zumal demokratische Strategen l(n)eidvoll bekunden, in punkto Technologie und Wählermarketing drei Jahre zurückzuliegen. ${ }^{98}$ Erst 2006 investierte die Republikanische Partei 15 Millionen US-Dollar, um ihre Daten zu aktualisieren. Bis zum Wahlabend wurden mit Hilfe von Voter Vault 24 Millionen US-Bürger direkt kontaktiert. ${ }^{99}$ Im Verbund mit der finanziellen Überlegenheit der Republikaner und ihrer engen Verflechtung mit Großindustrie und Lobbyisten trägt die Datenbank zu einem signifikanten Vorteil der Partei bei, der noch über Jahre hinweg spürbar sein wird.

\section{Fazit: Die Heterogenität der US-Konservativen als Hindernis auf dem Weg zur politischen Dominanz}

Die Antwort auf die Frage, ob die Bush-Präsidentschaft der Grundstein einer anhaltenden Dominanz der Republikaner sein kann oder gar, wie Tom Hamburger und Peter Wallsten meinen, auf ein „One Party Country“ hinausläuft, fällt ambivalent aus. Sicherlich sprechen viele Aspekte für eine solche Dominanz. Strukturell jedenfalls sind die Republikaner bestens für kommende Wahlen gerüstet. Dies ist nicht zuletzt ein Verdienst der Bush-Administration, die zweifelhafte Koalitionen wie das K Street-Projekt kompromisslos vorantrieb. Daneben haben Gesetze wie der No Child Left Behind Act dazu beigetragen, dass den Republikanern auch in wichtigen gesellschaftspolitischen Fragen eine hohe Problemlösungskompetenz zugetraut wird. Die klassisch libertäre Befürwortung von Bildungsgutscheinen spielt hier ebenfalls eine Rolle und stellt die Demokraten vor eine ernsthafte inhaltliche Zerreißprobe. Hinzu kommt die positive Haltung der Administration in Immigrationsfragen, die maßgeblich zur Popularität der Republikaner bei hispanischen Wählern beigetragen hat.

Doch hier beginnen die Zweifel an der Dominanz-These. Die Position der Republikanischen Partei in Einwanderungsfragen ist keineswegs homogen. Vielmehr ist ihr weltoffenes, „multikulturelles“ Image an einen scheidenden Präsidenten und dessen Inklusionspolitik geknüpft. Einflussreiche Parteimitglieder, unter anderem Ex-Präsidentschaftskandidat Mitt Romney, sprechen sich deutlich gegen die Einwanderungspolitik der gegenwärtigen Administration aus. Ob die Zugewinne bei den hispanischen Bevölkerungsteilen nachhaltig behauptet werden können, hängt also maßgeblich von den Positionen der Bush nachfolgenden Parteiführung ab. Und auch die auf eine kulturelle Transformation abzielende "ownership society“ ist auf halbem Wege stecken geblieben. Die US-Amerikaner in ein Volk von Aktienbesitzern umzuwandeln und sie damit quasi zu „natürlichen“ Parteigängern der Republikaner zu machen ist mit der Rentenreform auf ganzer Linie gescheitert. Die übrigen Reformen im Gesundheitswesen und Steuersystem sind lediglich als marginale Kursänderung zu bezeichnen. Dies gilt umso mehr, als die Steuersenkungen laut Gesetz im Jahre 2011 wieder zurückgenommen werden müssen.

98 Vgl. Tom Hamburger / Peter Wallsten, a.a.O., S. 153.

99 Vgl. Tom Hamburger / Peter Wallsten, Two parties far apart in turnout tactics too, in: The Los Angeles Times vom 6. November 2006, S. A1. 
Insgesamt überwiegen jedoch die Anzeichen, dass unter der Präsidentschaft George W. Bushs zumindest der Grundstein für eine anhaltende Dominanz der Republikaner gelegt wurde. Die strukturellen Gründe dafür wurden bereits angesprochen. Aber auch die gesellschaftspolitischen Positionen, so sehr diese auch innerhalb der Partei umstritten sein mögen, haben sich durchaus im Bewusstsein der Bevölkerung und in Teilen der Partei festgesetzt. Dazu gehört der neue Umgang mit Minderheiten genauso wie die breite und öffentliche Thematisierung kritischer Reformvorhaben. Daneben werden Religion und Moral künftig eine weiter steigende Bedeutung einnehmen - Themen, die traditionell den Republikanern in die Hände spielen. Festzuhalten bleibt jedoch auch, dass die Wahlen 2008 - viel mehr als die Zwischenwahlen 2006, die im Schatten des Irakkrieges und einer Reihe von Skandalen standen - den ersten Gradmesser auf dem Weg zum „One Party Country“ darstellen. Dann werden sich im Senat 22 Republikaner, aber nur zwölf Demokraten zur Wiederwahl stellen müssen, und der Kampf ums Weiße Haus wird weder gegen die polarisierende Hillary Clinton noch gegen den immer populäreren Barack Obama leicht werden. In diesem Kontext wird sich das tatsächliche Potenzial von Voter Vault, K Street-Projekt und „moderner" konservativer Sozialpolitik zeigen. 